\title{
CHIMGEN: a Chinese imaging genetics cohort to enhance cross- ethnic and cross-geographic brain research
}

\author{
Qiang $X u^{1} \cdot$ Lining Guo ${ }^{1}$ Jingliang Cheng ${ }^{2} \cdot$ Meiyun Wang ${ }^{3,4} \cdot$ Zuojun Geng $^{5} \cdot$ Wenzhen Zhu ${ }^{6} \cdot$ Bing Zhang $^{7}$. \\ Weihua Liao ${ }^{8,9}$. Shijun Qiu ${ }^{10}$. Hui Zhang ${ }^{11} \cdot$ Xiaojun $\mathrm{Xu}^{12} \cdot$ Yongqiang $\mathrm{Yu}^{13} \cdot \mathrm{Bo}^{12} \mathrm{Gao}^{14}$. Tong $\mathrm{Han}^{15,16}$.

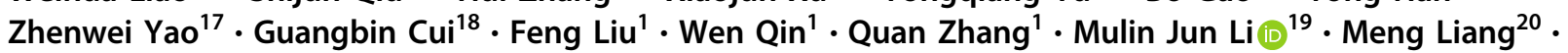 \\ Feng Chen $\mathbb{D}^{21}$. Junfang Xian ${ }^{22} \cdot$ Jiance $\mathrm{Li}^{23} \cdot$ Jing Zhang ${ }^{24} \cdot \mathrm{Xi}$-Nian Zuo $\mathbb{1}^{25,26} \cdot$ Dawei Wang ${ }^{27} \cdot$ Wen Shen $^{28}$. \\ Yanwei Miao ${ }^{29} \cdot$ Fei Yuan $^{30} \cdot$ Su Lui ${ }^{31,32} \cdot$ Xiaochu Zhang $\mathbb{1}^{33,34} \cdot \mathrm{Kai}^{\mathrm{X}} \mathrm{Xu}^{35,36} \cdot$ Long Jiang Zhang $^{37} \cdot$ Zhaoxiang Ye $^{38}$. \\ Chunshui $\mathrm{Yu}^{1,39} \cdot$ for the CHIMGEN Consortium
}

Received: 26 September 2018 / Revised: 21 November 2019 / Accepted: 27 November 2019 / Published online: 11 December 2019

(c) The Author(s) 2019. This article is published with open access

\begin{abstract}
The Chinese Imaging Genetics (CHIMGEN) study establishes the largest Chinese neuroimaging genetics cohort and aims to identify genetic and environmental factors and their interactions that are associated with neuroimaging and behavioral phenotypes. This study prospectively collected genomic, neuroimaging, environmental, and behavioral data from more than 7000 healthy Chinese Han participants aged 18-30 years. As a pioneer of large-sample neuroimaging genetics cohorts of non-Caucasian populations, this cohort can provide new insights into ethnic differences in genetic-neuroimaging associations by being compared with Caucasian cohorts. In addition to micro-environmental measurements, this study also collects hundreds of quantitative macro-environmental measurements from remote sensing and national survey databases based on the locations of each participant from birth to present, which will facilitate discoveries of new environmental factors associated with neuroimaging phenotypes. With lifespan environmental measurements, this study can also provide insights on the macro-environmental exposures that affect the human brain as well as their timing and mechanisms of action.
\end{abstract}

\section{Introduction}

Neuroimaging (intermediate) phenotypes reflecting the structural and functional properties of the human brain have been linked to human cognitive abilities and neuropsychiatric disorders (external phenotypes), and both intermediate and external phenotypes are precisely modulated by genetics, environments and their complex interactions [1, 2]. However, we know little about pathways from genetics and environments to neuroimaging phenotypes and

The members of the CHIMGEN Consortium are listed in the Supplementary file 1 .

Supplementary information The online version of this article (https:// doi.org/10.1038/s41380-019-0627-6) contains supplementary material, which is available to authorized users.

Chunshui $\mathrm{Yu}$

chunshuiyu@tmu.edu.cn

Extended author information available on the last page of the article then to external phenotypes. The associations between genetic factors and neuroimaging phenotypes have been investigated using neuroimaging genetics [3], initially by exploring the effects of a single nucleotide polymorphism (SNP) in small samples and eventually by identifying reliable genetic effects using genome-wide association studies (GWAS) in large samples [4, 5]. However, almost all available neuroimaging genetics cohorts include only Caucasian populations (Table 1), preventing us from identifying ethnic differences in genetic-neuroimaging associations. Although previous cohorts have included many microenvironmental factors, such as social economic status, early life events and lifestyle, few cohorts have included macroenvironmental factors derived from remote sensing and national survey databases, such as climate, air pollution, population density, and gross domestic product (GDP) per capita. The joint analyses of micro- and macroenvironmental variables will provide more information about environmental-neuroimaging associations and geneenvironment interactions on neuroimaging phenotypes $[6,7]$. Moreover, China has the largest populations in 
Table 1 Comparisons of major neuroimaging genetics cohorts $(N>2000$ with both genetic and neuroimaging data).

\begin{tabular}{|c|c|c|c|c|c|c|}
\hline Project name & $\begin{array}{l}\text { Number of } \\
\text { subjects }\end{array}$ & $\begin{array}{l}\text { Ethnic } \\
\text { populations }\end{array}$ & $\begin{array}{l}\text { Age } \\
\text { range (year) }\end{array}$ & Diagnosis & Prominent features & $\begin{array}{l}\text { Assessments of data } \\
\text { quality }^{\mathrm{a}}\end{array}$ \\
\hline CHIMGEN & $N>7000$ & $\begin{array}{l}\text { Chinese } \\
\text { Han only }\end{array}$ & $18-30$ & Healthy & $\begin{array}{l}\text { The largest prospective neuroimaging genetics cohort of } \\
\text { Chinese Han adults with lifespan natural and socioeconomic } \\
\text { environmental measurements obtained from remote sensing } \\
\text { and national survey databases }\end{array}$ & $\begin{array}{l}\text { Brain imaging data }(+++) \text { : } \\
3 \text { modalities for all subjects; } \\
2 \text { for } 2 / 3 \text { subjects } \\
\text { Genetic data }(++++) \text { : } \\
\text { genomic data } \\
\text { Environment data }(+++ \\
+) \text { : more than a hundred of } \\
\text { quantitative measures } \\
\text { Behavioral data }(++++) \text { : } \\
\text { dozens of measures }\end{array}$ \\
\hline UK Biobank & $N>30,000$ & $\begin{array}{l}\text { Most } \\
\text { Caucasian }\end{array}$ & $\begin{array}{l}40-69 \text { at } \\
\text { baseline }\end{array}$ & Mixed & $\begin{array}{l}\text { The largest prospective longitudinal imaging genetics } \\
\text { cohort of adults in the world }\end{array}$ & $\begin{array}{l}\text { Brain imaging }(++++) \text { : } 5 \\
\text { imaging modalities for most } \\
\text { subjects } \\
\text { Genetic data }(++++) \text { : } \\
\text { genomic data } \\
\text { Environment data }(++) \text { : } \\
\text { dozens of measures } \\
\text { Behavioral data }(++++) \text { : } \\
\text { dozens of measures }\end{array}$ \\
\hline ENIGMA & $N>50,000$ & $\begin{array}{l}\text { Most } \\
\text { Caucasian }\end{array}$ & $3.3-91$ & Mixed & $\begin{array}{l}\text { The largest imaging genetics pooling dataset included more } \\
\text { than } 50 \text { currently available datasets with both imaging and } \\
\text { genetic data }\end{array}$ & $\begin{array}{l}\text { Brain imaging }(++) \text { : only } \\
\text { structural imaging for all } \\
\text { subjects } \\
\text { Genetic data }(++++) \text { : } \\
\text { genomic data } \\
\text { Environment data }(-) \text { : no } \\
\text { measure } \\
\text { Behavioral data }(++) \text { : no } \\
\text { measure but with diagnostic } \\
\text { information }\end{array}$ \\
\hline $\mathrm{ABCD}$ & $N=11,875$ & $\begin{array}{l}\text { Most } \\
\text { American } \\
\text { African and } \\
\text { Caucasian }\end{array}$ & $\begin{array}{l}9-10 \text { at } \\
\text { baseline }\end{array}$ & $\begin{array}{l}\text { Relatively } \\
\text { healthy }\end{array}$ & $\begin{array}{l}\text { The largest prospective longitudinal imaging genetics } \\
\text { cohort of children to explore adolescent brain development }\end{array}$ & $\begin{array}{l}\text { Brain imaging }(++++): 4 \\
\text { modalities for most subjects } \\
\text { Genetic data }(++++) \text { : } \\
\text { genomic data } \\
\text { Environment data }(++) \text { : } \\
\text { dozens of measures } \\
\text { Behavioral data }(++++) \text { : } \\
\text { dozens of measures }\end{array}$ \\
\hline IMAGEN & $N=2000$ & $\begin{array}{l}\text { Most } \\
\text { Caucasian }\end{array}$ & $14-22$ & $\begin{array}{l}\text { Relatively } \\
\text { healthy }\end{array}$ & $\begin{array}{l}\text { The first prospective longitudinal imaging genetics cohort } \\
\text { of adolescence to investigate the risk for mental disorders }\end{array}$ & $\begin{array}{l}\text { Brain imaging }(++++): 4 \\
\text { modalities for most subjects } \\
\text { Genetic data }(++++) \text { : } \\
\text { genomic data } \\
\text { Environment data }(++) \text { : } \\
\text { dozens of measures } \\
\text { Behavioral data }(++++) \text { : } \\
\text { dozens of measures }\end{array}$ \\
\hline ADNI & $N>2000$ & $\begin{array}{l}\text { Most } \\
\text { Caucasian }\end{array}$ & $55-90$ & $\begin{array}{l}\mathrm{AD}, \mathrm{MCI} \\
\text { and normal } \\
\text { controls }\end{array}$ & $\begin{array}{l}\text { The largest prospective longitudinal imaging genetics } \\
\text { cohort of elderly people to define the progression of } \mathrm{AD}\end{array}$ & $\begin{array}{l}\text { Brain imaging }(+++) \text { : } 5 \\
\text { modalities but not collected } \\
\text { from all subjects } \\
\text { Genetic data }(++++) \text { : } \\
\text { genomic data } \\
\text { Environment data }(+) \text { : a } \\
\text { few measures } \\
\text { Behavioral data }(++++) \text { : } \\
\text { dozens of measures }\end{array}$ \\
\hline
\end{tabular}

$A B C D$ Adolescent Brain Cognitive Development, $A D$ Alzheimer's disease, ADNI Alzheimer's Disease Neuroimaging Initiative, $C H I M G E N$ Chinese Imaging Genetics, ENIGMA Enhancing Neuro Imaging Genetics Through Meta-Analysis, IMAGEN imaging genetics, $M C I$ mild cognitive impairment

${ }^{a}$ Notes: The seven major imaging modalities included structural imaging, susceptibility weighted imaging (SWI), diffusion tensor imaging (DTI), artery spin labeling (ASL), task fMRI, resting-state fMRI, and positron emission tomography (PET). For each kind of data, the number of + signs indicates the subjective data availability, which includes two factors: the richness of the variables and the number of participants with data on these variables

the world and has experienced dramatic changes in its macro-environments in recent decades, making the Chinese population more suitable for identifying macroenvironmental factors associated with neuroimaging phenotypes.
Although the China Brain Project, which covers basic neuroscience, translational research, and brain-inspired intelligence, is being developed [8], there are no available large-scale Chinese neuroimaging genetics data. In this context, the Chinese Imaging Genetics (CHIMGEN) study 
was designed to collect genomic, environmental, neuroimaging, and behavioral data from a large number of Chinese participants to enhance neuroimaging genetics research in different ethnic populations and geographic locations. Compared with currently available large-scale neuroimaging genetics studies (Table 1), the CHIMGEN study includes the only cohort of non-Caucasian participants and has collected hundreds of macro-environmental measurements in addition to micro-environmental measurements. These comprehensive multiscale data can fill the gap in our understanding of how environmental factors, and their interaction with genetic factors can affect the human brain and consequently affect behavior by using effective dimension reduction or feature selection techniques [9-11].

\section{The CHIMGEN study}

The CHIMGEN study (chimgen.tmu.edu.cn) was approved by the local ethics committee, and written informed consent was obtained from each participant. The aim of this study was to collect genomic, neuroimaging, environmental, and behavioral data from 10,000 healthy Chinese Han participants aged 18-30 years in 30 research centers from 21 mainland cities in China. To date, we have recruited more than 7000 participants, becoming the largest and most integrative Chinese neuroimaging genetics cohort. The detailed inclusion and exclusion criteria as well as the methods and procedures for screening; genotyping; blood sample collection; and behavioral, environmental, and neuroimaging data acquisition are described in the standardized operation procedures (SOPs) of the CHIMGEN study (Supplementary file 2). The detailed quality control procedures for personal information; blood samples; GWAS; and behavioral, environmental, and neuroimaging assessments are elaborated in the quality control manual of the CHIMGEN study (Supplementary file 3). Since the CHIMGEN study is ongoing, the following description of the CHIMGEN cohort was based on the data of only 5819 participants who had undergone comprehensive quality assessments.

\section{Sampling strategies}

All participants were recruited by advisements posted in colleges and communities. The number of participants in each center depends on the available resources (researchers, funds, scanners, etc.) of the center. The recruited participants were not solely from the city or province of the participating centers. These samples are not used to represent populations (epidemiological samples), but to investigate biological mechanisms. Their epidemiological relevance needs to be investigated in subsequent studies.

\section{Recruitment distribution}

The 5819 participants were recruited from 29 centers. The recruitment distribution of these participants across centers is shown in Fig. 1a. Eighteen of the 29 centers recruited more than 100 participants. The largest center recruited 1307 participants and the smallest center recruited 54 participants.

\section{Quality control for MR scanners}

For each MR scanner, two phantoms were used to assess the imaging quality of the scanner. Specifically, an American College of Radiology MRI phantom was used to assess the functioning of the MR scanner, including geometric distortion, slice positioning and thickness accuracy, high contrast spatial resolution, intensity uniformity, ghosting artefacts and low contrast object detectability. A custom phantom $[12,13]$ was used to evaluate temporal stability during a functional MRI acquisition. Moreover, two healthy volunteers were scanned at all centers to assess the consistency of the MRI data acquired by different MR scanners. The effects of scanners on common MRI measures (gray matter volume (GMV), regional homogeneity (ReHo) and fractional anisotropy (FA)) are shown in Supplementary Fig. 1. These measures showed high consistency for MRI data acquired by the same type of MR scanner with the same scan parameters; however, there were visible differences for MRI data acquired by different types of MR scanners. For the latter, a meta-analysis of the results derived from MR data from different scanners may be a practical method to reduce the bias caused by MR scanner types.

\section{First-step quality assessments of the neuroimaging data}

All 5819 participants were included in the first-step quality assessments of the neuroimaging data: 23 participants were excluded for metal artefacts, 1 for brain atrophy and 1 for excessively large ventricle. The remaining 5794 participants were included in the following quality control and statistics.

\section{Genotyping and quality control}

A high-throughput genotyping chip designed for the Asian population (Illumina Asian screening array chip) with 700,000 sampling SNPs was used for genome-wide genotyping. Although all 5794 participants had blood samples, only 4885 participants have been genotyped thus far. After excluding two sex mismatching samples, nine duplicated or related samples, 29 samples with extreme heterozygosity and one sample with divergent ancestry (Supplementary 


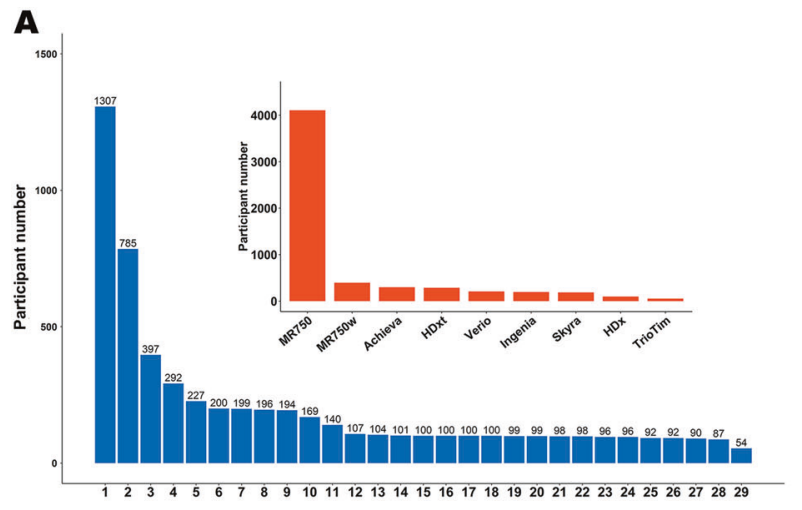

C
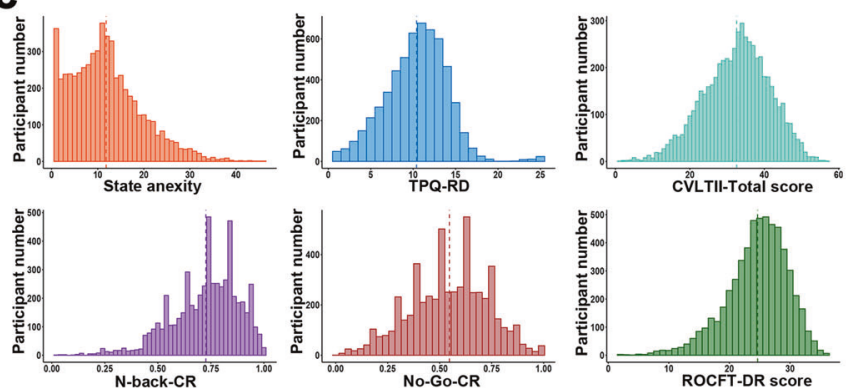

Fig. 1 Recruitment and neuroimaging, behavioral, and environmental characteristics. a The main graph shows the numbers of participants recruited by each of the 29 centers. The insertion shows the numbers of participants recruited using each type of scanner. $\mathbf{b}$ The mean parameter maps of the gray matter volume (GMV), regional homogeneity (ReHo), fractional anisotropy (FA), mean diffusivity (MD), mean kurtosis (MK), and cerebral blood flow (CBF). c Data distribution of the representative behavioral assessments. CVLT IITotal score, the total number of correct recalls over the five learning

Fig. 2), 4844 participants (99.16\%) passed the quality control for the genetic data. It should be noted that the following quality assessments $(n=5753)$ also included 909 participants without genotyping results.

\section{Neuroimaging data and quality control}

Neuroimaging data were acquired by nine types of 3.0Tesla MRI scanner (Supplementary Fig. 3). Structural MRI (sMRI), diffusion tensor imaging (DTI) and restingstate functional MRI (rs-fMRI) data were acquired in all centers, and diffusion kurtosis imaging (DKI) and arterial spin labeling (ASL) data were acquired in 16 centers. The numbers of participants whose MRI data were acquired by each type of MRI scanner are shown in the insertion of Fig. 1a. The MRI data of $4045(70.31 \%)$ of the 5753 participants were acquired by the MR 750 scanners. For each type of MRI scanner, the voxel-level maps of GMV calculated based on sMRI data, ReHo calculated based on rs-fMRI data, and FA and mean diffusivity (MD) calculated based on DTI data averaged across all qualified
B

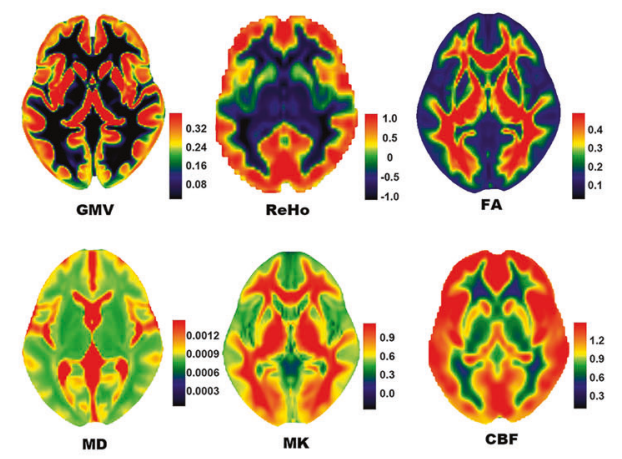

D

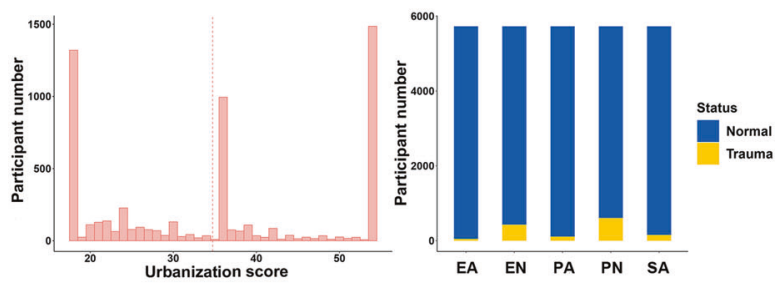

trials of the word list $\mathrm{A}$ in the version 2 of the California verbal learning test; N-back-CR, the correct rate of the 3-back task in the Nback task; No-Go-CR, the correct rate of the No-Go task in the Go/ No-Go task; ROCFT-DR score, the score of delayed recall of the ReyOsterrieth complex figure test; TPQ-RD, reward dependence of tridimensional personality questionnaire. d Data distribution of the representative paper-based environmental assessments. EA emotional abuse, EN emotional neglect, PA physical abuse, PN physical neglect, and SA sexual abuse. participants are shown in Supplementary Fig. 4. All types of scanner showed similar and symmetrical spatial distribution of the GMV, FA and MD, and 8/9 types of scanner showed similar and symmetrical spatial distribution of ReHo with the GE Signa HDx which showed asymmetric spatial distribution of the ReHo map, especially in posterior brain regions, being the only exception (Supplementary Fig. 4C). Therefore, the rs-fMRI data of the 97 participants acquired by the GE Signa HDx were excluded from this study.

The quality control results of the neuroimaging data $(n=5753)$ are shown in Supplementary Fig. 5. In the 5753 participants, there were $5743(99.83 \%)$ participants with qualified sMRI data, 5507 (95.72\%) with qualified rs-fMRI data, and 5750 (99.95\%) with qualified DTI data. In the 3619 participants with DKI data, 3610 (99.75\%) participants passed the quality control. In the 4108 participants with ASL data, all participants passed the quality control. Based on these MRI data, thousands of neuroimaging variables could be calculated. For example, the average maps of the GMV of the 5743 participants, the 
ReHo of the 5507 participants, the FA and MD of the 5750 participants, the mean kurtosis (MK) calculated based on DKI data of the 3610 participants, and the cerebral blood flow (CBF) calculated based on ASL data of the 4108 participants are shown in Fig. 1b. All of these parameter maps showed a symmetrical spatial distribution in the brain.

\section{Quality control for behavioral and paper-based environmental data}

The preliminary quality control results for behavioral and paper-based environmental data of the 5753 participants are shown in Supplementary Fig. 6. In the 5753 participants, 8 participants were excluded for the loss of almost all behavioral and paper-based environmental data. In the remaining 5745 participants, $5723(99.48 \%)$ participants with qualified Beck depression inventory (BDI- II) data, 5722 (99.46\%) with qualified state and trait anxiety inventory (STAI) data, 5728 (99.57\%) with qualified tridimensional personality questionnaire (TPQ) data, $5688(98.87 \%)$ with qualified California verbal learning test (CVLT-II) data, 5619 (97.67\%) with qualified symbol digit modalities test (SDMT) data, 5640 (98.04\%) with qualified Rey-Osterrieth complex figure test (ROCFT) data, 5578 (96.96\%) with qualified N-back task data, $5536(96.23 \%)$ with qualified Go/No-Go task data, 5616 (97.62\%) with qualified balltossing game data, 5639 (98.02\%) with qualified ultimatum game (UG) data, 5733 (99.65\%) with qualified urbanization score data, and 5728 (99.57\%) with qualified childhood trauma questionnaire (CTQ) data.

The data distributions of the representative behavioral variables are demonstrated in Fig. 1c and those of the representative paper-based environmental variables are shown in Fig. 1d. Although some variables do not follow a normal distribution, the relatively wide range of values indicates good discriminative power across participants.

\section{Sample characteristics}

The demographic characteristics of the 5745 participants with relatively complete assessments are shown in Table 2 . This study included 3718 females and 2027 males. Their ages ranged from 18 to 30 years, with a mean \pm standard deviation (SD) of $23.7 \pm 2.4$ years. Their years of education ranged from 9 to 24 years, with a mean \pm SD of $16.8 \pm 1.9$ years. Their heights ranged from 146 to $197 \mathrm{~cm}$, with a mean \pm SD of $166.4 \pm 7.9 \mathrm{~cm}$. Their weights ranged from 23 to $114 \mathrm{~kg}$, with a mean \pm SD of $58.8 \pm 10.7 \mathrm{~kg}$. Their body mass indices (BMI) ranged from 10.8 to 38.5 , with a mean \pm SD of $58.8 \pm 10.7$. Most of these participants were unmarried $(n=5550)$, with only 195 married.
Sex-specific demographic, behavioral, and paperbased environmental statistics

The sex-specific demographic, behavioral and paper-based environmental statistics of the 5745 participants with relatively complete assessments are shown in Table 2. Although most of these variables show significant differences $(P<$ 0.05 ) between male and female participants, the effect sizes were generally very small except for sex differences in height $(|r|=0.74$, large effect), weight $(|r|=0.67$, large effect), and BMI $(|r|=0.39$, medium effect $)$.

\section{Quantitative environmental variables derived from remote sensing and national survey databases}

In this study, we recorded the precise residential location of each participant in each year from birth to present. In the 5745 participants who passed the initial quality controls for the neuroimaging, behavioral and genetic data, 5723 participants $(99.62 \%)$ provided both current and birthplace (Fig. 2a) residential locations; however, only 3979 participants $(69.26 \%)$ provided lifetime migration information (Fig. 2b). Based on remote sensing and national survey databases, we obtained hundreds of macro-environmental measurements for each participant. Some representative macro-environmental variables at birth (Fig. 2c) and their lifetime changes are shown in Fig. 2d.

\section{Future plans of the CHIMGEN study}

In the future, the CHIMGEN consortium will complete the following tasks: (a) further recruit at least 3000 participants to reach the goal of 10,000 qualified participants; (b) simultaneously obtain the genomic, epigenomic, and transcriptomic data of $\sim 700$ participants; (c) collect 2000-3000 patients with major mental disorders; and (d) develop the CHIMGEN cohort into a longitudinal cohort by recalling the participants at a later time.

\section{Data sharing policy}

We would like to share all CHIMGEN data (including the genetic, environmental, neuroimaging and behavioral data) with other scientific communities according to the laws and regulations of the Chinese government. All the raw data of the CHIMGEN study can be accessed via collaboration with the CHIMGEN consortium. The summary statistics of the CHIMGEN data can be freely accessed via a formal application. A detailed scheme for sharing the CHIMGEN data can be found on our website (chimgen.tmu.edu.cn) and in Supplementary file 4. 
Table 2 Sex-specific demographic, behavioral and environmental data $(n=5745)$.

\begin{tabular}{|c|c|c|c|c|c|c|}
\hline \multirow[t]{2}{*}{ Items } & \multirow[t]{2}{*}{ Total $($ mean $\pm \mathrm{SD})$} & \multirow[t]{2}{*}{ Males $($ mean $\pm \mathrm{SD})$} & \multirow[t]{2}{*}{ Females $($ mean $\pm \mathrm{SD})$} & \multicolumn{3}{|c|}{ Gender differences } \\
\hline & & & & $Z / \chi^{2}$ value & $P$-value & Effect size \\
\hline Age (years) & $23.7 \pm 2.4(n=5745)$ & $23.6 \pm 2.6(n=2027)$ & $23.7 \pm 2.3(n=3718)$ & -2.77 & $5.58 \times 10^{-3}$ & -0.04 \\
\hline Years of education & $16.8 \pm 1.9(n=5745)$ & $16.5 \pm 1.9(n=2027)$ & $16.9 \pm 1.9(n=3718)$ & -8.18 & $2.74 \times 10^{-16}$ & -0.11 \\
\hline Marital status (No/Yes) & $5550 / 195(n=5745)$ & $1941 / 86(n=2027)$ & $3609 / 109(n=3718)$ & 6.88 & $8.73 \times 10^{-3}$ & 0.03 \\
\hline Height $(\mathrm{cm})$ & $166.4 \pm 7.9(n=5745)$ & $174.5 \pm 5.7(n=2027)$ & $162.0 \pm 5.0(n=3718)$ & -56.35 & $<1.15 \times 10^{-190}$ & -0.74 \\
\hline Weight $(\mathrm{Kg})$ & $58.8 \pm 10.7(n=5745)$ & $68.5 \pm 10.1(n=2027)$ & $53.6 \pm 6.6(n=3718)$ & -51.12 & $<1.15 \times 10^{-190}$ & -0.67 \\
\hline BMI & $21.1 \pm 2.6(n=5745)$ & $22.5 \pm 2.8(n=2027)$ & $20.4 \pm 2.2(n=3718)$ & -29.45 & $1.15 \times 10^{-190}$ & -0.39 \\
\hline TPQ-NS & $13.8 \pm 4.5(n=5728)$ & $13.0 \pm 4.3(n=2019)$ & $14.2 \pm 4.5(n=3709)$ & -9.48 & $2.66 \times 10^{-21}$ & -0.13 \\
\hline BDI-II & $3.37 \pm 4.41(n=5723)$ & $3.01 \pm 4.06(n=2017)$ & $3.56 \pm 4.58(n=3706)$ & -4.25 & $2.19 \times 10^{-5}$ & -0.06 \\
\hline State anxiety & $30.8 \pm 7.6(n=5722)$ & $30.2 \pm 7.5(n=2018)$ & $31.2 \pm 7.6(n=3704)$ & -5.39 & $7.20 \times 10^{-8}$ & -0.07 \\
\hline CVLT II-total score & $55.6 \pm 8.8(n=5703)$ & $54.1 \pm 9.1(n=2014)$ & $56.4 \pm 8.6(n=3689)$ & -9.06 & $1.32 \times 10^{-19}$ & -0.12 \\
\hline ROCFT-DR score & $24.7 \pm 5.0(n=5643)$ & $24.8 \pm 5.0(n=1989)$ & $24.6 \pm 5.0(n=3654)$ & -1.46 & 0.15 & -0.02 \\
\hline SDMT score & $69.6 \pm 12.7(n=5619)$ & $68.8 \pm 13.1(n=1989)$ & $70.0 \pm 12.5(n=3630)$ & -4.12 & $3.76 \times 10^{-5}$ & -0.05 \\
\hline N-back-CR & $0.73 \pm 0.16(n=5578)$ & $0.74 \pm 0.15(n=1973)$ & $0.72 \pm 0.16(n=3605)$ & -5.88 & $4.18 \times 10^{-9}$ & -0.079 \\
\hline No-Go-CR & $0.55 \pm 0.19(n=5536)$ & $0.58 \pm 0.19(n=1957)$ & $0.53 \pm 0.18(n=3579)$ & -10.05 & $9.48 \times 10^{-24}$ & -0.14 \\
\hline UG-AR & $0.76 \pm 0.32(n=5639)$ & $0.78 \pm 0.31(n=1989)$ & $0.74 \pm 0.33(n=3650)$ & -4.08 & $4.54 \times 10^{-5}$ & -0.05 \\
\hline BTG-ACC & $0.80 \pm 0.19(n=5616)$ & $0.81 \pm 0.19(n=1977)$ & $0.79 \pm 0.19(n=3639)$ & -3.82 & $1.34 \times 10^{-4}$ & -0.05 \\
\hline Urbanization score & $34.7 \pm 13.9(n=5733)$ & $35.2 \pm 14.0(n=2022)$ & $34.5 \pm 13.9(n=3711)$ & -1.91 & 0.06 & -0.03 \\
\hline CTQ-EN & $8.19 \pm 4.17(n=5728)$ & $8.01 \pm 3.98(n=2022)$ & $8.28 \pm 4.26(n=3706)$ & -1.45 & 0.15 & -0.02 \\
\hline
\end{tabular}

Notes: Only one representative measure of each behavioral or environmental assessment is shown in this table. The sample sizes of the behavioral and environmental assessments are different across measures because only qualified participants are included in the statistical analysis. The effect sizes for categorical variables are evaluated by $\Phi$ and those for continuous variables are evaludated with Mann-Whitney and Wilcoxon nonparametric tests using $r$. Cohen's guidelines for effect size are that a large effect is $>0.5$, a medium effect is between 0.3 and 0.5 , and a small effect is between 0.1 and 0.3

$B D I-I I$ beck depression inventory II, BMI body mass index, BTG-ACC the total correct rate in the ball-tossing game, $C T Q-E N$ the emotional neglect score of childhood trauma questionnaire, CVLT II-Total score the total number of correct recalls over the five learning trials of the word list $\mathrm{A}$ in the version 2 of the California verbal learning test, $\mathrm{N}$-back-CR the correct rate of the 3-back task in the N-back task, No-Go-CR the correct rate of the No-Go task in the Go/ No-Go task, ROCFT-DR score the score of delayed recall of the Rey-Osterrieth complex figure test, SDMT symbol digit modalities test, $T P Q-N S$ novelty-seeking of tridimensional personality questionnaire, $U G$ - $A R$ the ratio of participants who accept the $1: 9$ allocation schemes in situation 1 of the ultimate game. In this situation, if the participant accepts the plan, the proposer and the participant will divide the money according to this plan. If the participant rejects the plan, neither of them gets the money

\section{Discussion}

With genomic, environmental, neuroimaging, and behavioral data, the CHIMGEN study will help answer the following scientific questions about the associations between genetic and environmental factors on one hand and brain and cognitive phenotypes on the other hand.

\section{Cross-ethnic differences in genetic-neuroimaging associations}

Although GWAS analyses have identified many genetic variants associated with cognitive and neuropsychiatric phenotypes [14-16], we know little about the genetic variants associated with neuroimaging phenotypes. The most substantial obstacle for neuroimaging genetics studies is the time and economic cost of collecting high-quality neuroimaging data in a large sample (e.g., 10,000 participants).
Fortunately, European and American countries have launched several large-scale neuroimaging genetics studies $(n>$ 2000) (Table 1), such as the Alzheimer Disease Neuroimaging Initiative (ADNI) [17, 18], Imaging Genetics (IMAGEN) [19], Enhancing Neuroimaging Genetics through Meta-analysis (ENIGMA) [20], UK Biobank (UKBB) [21], and Adolescent Brain Cognitive Development (ABCD) [22]. These studies aim to identify reliable genetic variants associated with neuroimaging phenotypes and to discover new biomarkers for neuropsychiatric disorders. However, the majority of the participants included in these cohorts are Caucasian.

Ethnic differences have been reported in the allele frequencies of SNPs [23-25], linkage disequilibrium and polygenic risk scores [26], genetic susceptibilities for neuropsychiatric disorders [27], and neuroimaging phenotypes [28-30]. In addition to environmental factors, genetic factors are the main causes for ethnic differences in 
A
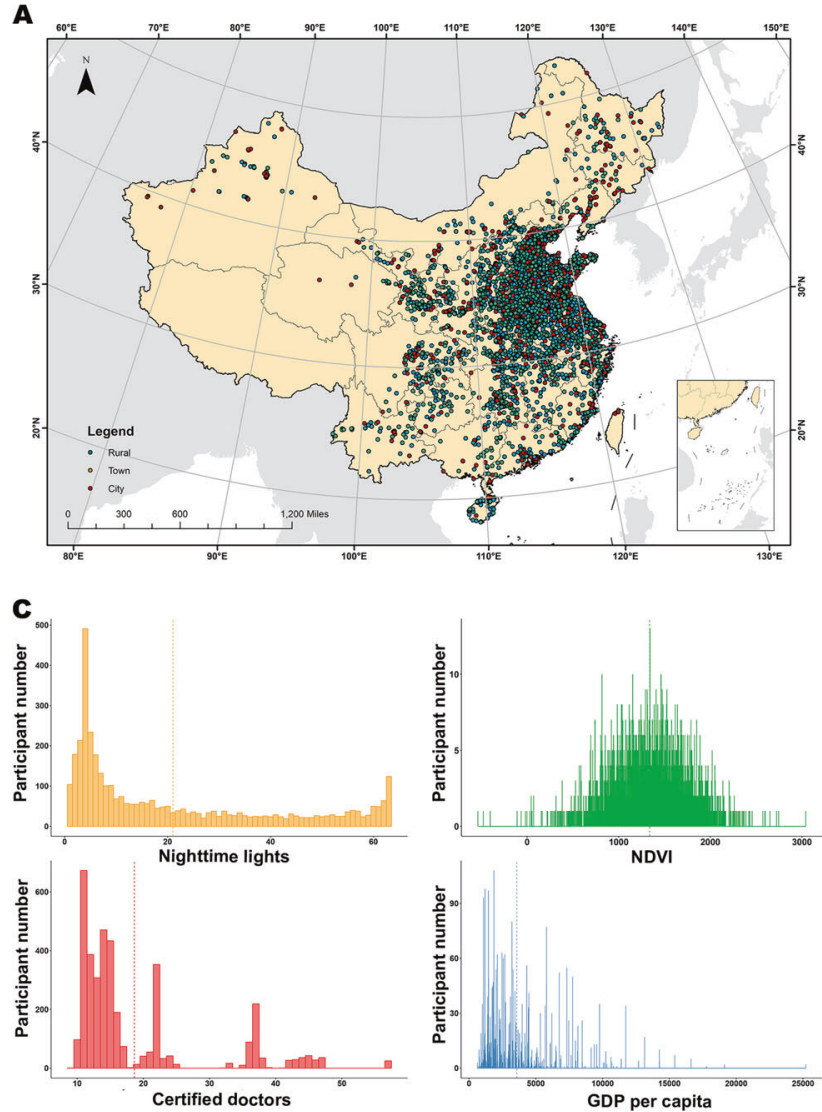

Fig. 2 Environmental variables derived from remote sensing and national survey data. a Geographic location of each participant's birthplace $(n=5723)$. Blue dots indicate rural area, green dots indicate towns, and red dots indicate cities. b The migration map of participants $(n=3979)$. Red dots indicate current places of residence, and green dots indicate birthplaces. Gray lines connect the birthplaces and current places of residence of a given participant. $\mathbf{c}$ Data distribution of

neuroimaging phenotypes because of their high heritability [31-33]. However, the common and specific genetic variants associated with neuroimaging phenotypes of different ethnic populations remain unknown, because there is no available large-scale neuroimaging genetics cohort of nonCaucasian individuals. From this perspective, the CHIMGEN data will provide an opportunity to discover ethnic differences in neuroimaging-related genetic variants between Chinese and Caucasian participants.

Although it is clinically important to identify genetic associations with neuroimaging markers of neuropsychiatric disorders [34-38], it is also critical to identify geneticneuroimaging associations in normal populations to better understand how genetic variants cause brain structural and functional impairments in neuropsychiatric disorders. However, none of the large-scale neuroimaging genetics studies $(n>2000)$ have included a sufficient number of healthy adults aged 18-30 years (Table 1), an age window during which human brains and their functions are minimally influenced by the confounding factors of
B
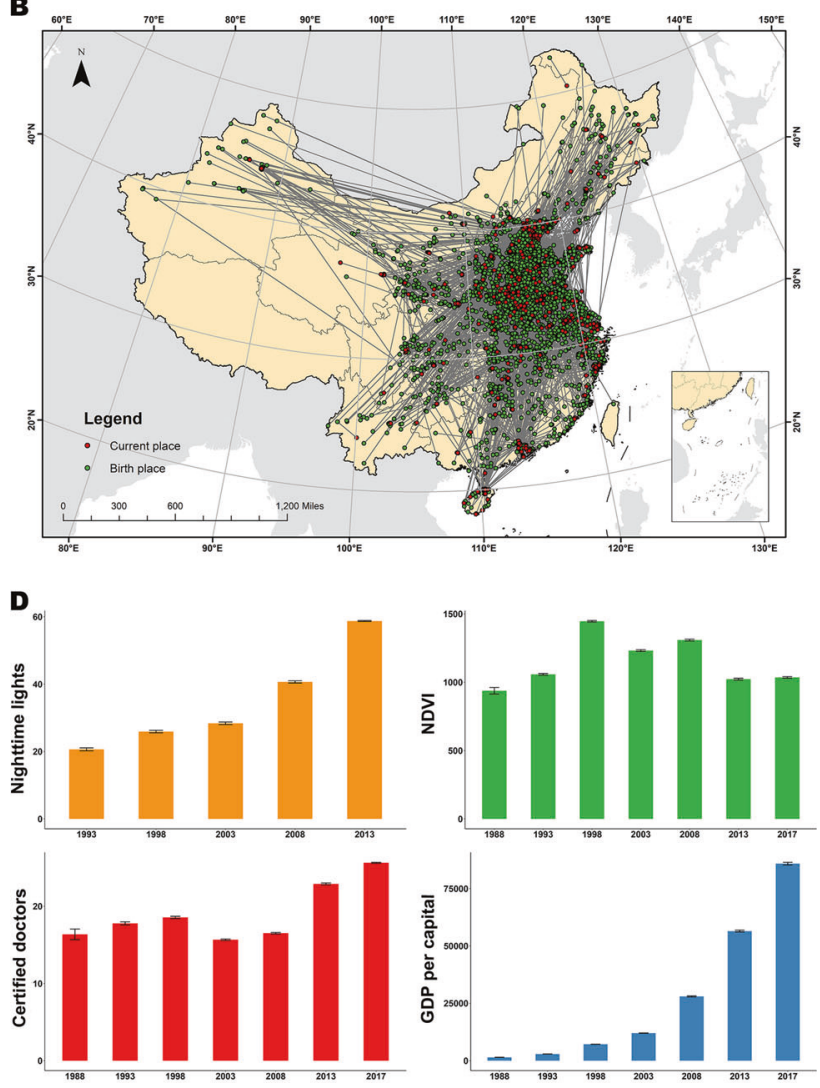

the representative environmental variables in the birth year or the year nearest to the birth year. Certified doctors is the number of certified doctors per 10,000 persons. NDVI, normalized difference vegetation index, and GDP, gross domestic product. d Longitudinal changes of the representative environmental variables in selected years. The value in each column is shown as the mean $\pm \mathrm{SE}$.

development and ageing [39]. Thus, the CHIMGEN study of 7000 healthy adults between $18-30$ years is suitable for investigating genetic-neuroimaging associations in unaged mature brains.

\section{Environmental factors associated with neuroimaging phenotypes}

One unique aspect of the CHIMGEN study is the collection of hundreds of macro-environmental measurements from satellite images and national survey databases. Compared with micro-environmental assessments based on questionnaire and self-report data, remote sensing, and national survey data can provide many new quantitative macroenvironmental assessments. For example, we can obtain quantitative environmental measurements of landform and topography, urbanization, climate, and air quality of the living places of each participant based on remote sensing data [40-43], and those of economy, urbanization, living condition, healthcare, and education of the living places of 
the participant based on national survey databases (data. stats.gov.cn/english/). Associations between neuroimaging phenotypes and most of these macro-environmental measurements have not been explored, and they may provide us with an opportunity to discover new environmental factors related to neuroimaging phenotypes. The feasibility of using macro-environmental measurements derived from remote sensing and national survey databases to discover new environmental factors associated with the human brain and behavioral phenotypes has been tested in pilot studies. For example, the green space assessed by the normalized difference vegetation index (NDVI) based on remote sensing data has been linked to human health [44, 45], and the lifelong exposure to greenness has been associated with GMV differences in children [7]. In addition, several macro-environmental measurements derived from national survey databases, such as population density, local GDP per capita, medical supply, and educational resources have also been associated with human health [46-48].

More importantly, with the precise lifelong residential locations of each participant, we can obtain the macroenvironmental measurements of each participant in each year from birth to present, from which we can estimate the cumulative exposure of environmental risk factors throughout the lifespan or during a period of interest. The detailed lifelong environmental data of the CHIMGEN study will help determine the macro-environmental exposures that affect the structural and functional properties of the human brain as well as their timing and mechanisms of action.

\section{Genome-wide by environment interactions on neuroimaging phenotypes}

Most neuropsychiatric disorders have a multifactorial etiology and emerge through the interplay of genetic and environmental factors [49]. Similarly, the structural and functional architectures of the human brain are also modulated by both factors [50], and gene-environment interactions may explain the missing heritability of certain phenotypes [51]. Candidate-gene approaches have been used extensively to explore gene-environment interactions. For example, the serotonin transporter promoter polymorphism interacts with stressful life events to increase the risk of depression [52]. However, candidategene approaches are criticized for oversimplifying the genetic substrates of these complex phenotypes since a single genetic variant minimally contributes to these phenotypes. The PRS integrates many genetic variants of the genome and is a better representation of genetic risk than single variants by having a much larger effect $[53,54]$. Indeed, considering the combination of PRS and childhood trauma can improve the ability to predict depression $[55,56]$. Genome-wide by environment interactions have been used to unbiasedly explore the effects of gene-environment interactions on depression [57]. However, the lack of large dataset simultaneously with genome-wide genetic data, objective environmental assessments and neuroimaging data has prevented investigations of genome-wide by environment interactions on neuroimaging phenotypes. In this context, the CHIMGEN study has rich genomic, environmental, and neuroimaging measurements of 7000 participants, and is particularly suited to investigate genome-wide by environment interactions on human neuroimaging phenotypes.

\section{Gene (environment)-brain-behavior pathways}

In contrast to many studies focusing on pairwise correlations of genetic variants, environmental factors, neuroimaging measures, and cognitive or neuropsychiatric phenotypes, only a few studies have explored biological pathways from genes and environment to brain structure and function and ultimately to cognition and symptoms [58-60]. These studies have been primarily conducted using candidate-gene approaches and small samples, and they have been criticized based on the minimal effect size of a single variant and their lack of statistical power. In view of polygenic profiles of neuroimaging and cognitive phenotypes [61, 62], genomic data should be integrated to identify normal and abnormal gene-brain-behavior pathways. Since environmental factors alone and gene-environment interactions affect neuroimaging and cognitive phenotypes $[6,63,64]$, it is important to identify the environmental factors associated with these phenotypes, which would help better guide clinical practice to address these adverse environmental factors. Furthermore, it is also critical to investigate how gene-environment interactions affect brain structure and function and then influence normal cognitive functions and brain disorders. By gathering genomic, environmental, neuroimaging, and cognitive data, the CHIMGEN project is ideally suited to explore the normal pathways of gene (environment)-brain-cognition.

\section{Comprehensive understanding of human cognitive functions with multiscale data}

The human brain is the most complex system in the world, and even the simplest cognitive task requires an efficient cooperation of multiscale neural elements $[65,66]$. Thus, human cognitive function can be understood only by integrating multimodal data at different scales, e.g., genomic, epigenomic, transcriptomic, and proteomic data at the microscale, neural circuit, and neuronal activity data at the mesoscale, and neuroimaging data at the macroscale. In addition to establishing reliable correlations between 
multiscale features and cognitive functions, it is also critical to identify causal linkages between these features to discover the causal pathways from the microscale to the mesoscale then to the macroscale and ultimately to cognition [8]. With genomic, transcriptomic, epigenomic, neuroimaging, and cognitive data obtained from 700 participants, the CHIMGEN study can be used to establish associations between microscale genetic variants and macroscale neuroimaging phenotypes, and then the functions of the identified genetic variants can be explored and validated at the cellular level [67] and in animal models [68] using gene editing techniques. One can also try to identify causal links among findings from different scales by integrating currently available multiscale neurobiological datasets and state-of-the-art bioinformatics.

\section{Associations with major neuropsychiatric disorders}

Many neuropsychiatric disorders are associated with genetic and environmental factors and their interactions [69]. We have identified many risk factors for major neuropsychiatric disorders, but the underlying mechanisms remain largely unknown. Taking neuroimaging measures as intermediate phenotypes, researchers could explore how these factors increase the risk for neuropsychiatric disorders by investigating the effects of these factors on neuroimaging measures in healthy subjects. For example, the CHIMGEN data can be used to investigate the effects of a single or integrated genetic and/or environmental risk factor(s) for neuropsychiatric disorders on neuroimaging phenotypes in healthy individuals. Moreover, we can identify new genetic or environmental risk factors that significantly affect neuroimaging markers of neuropsychiatric disorders.

\section{Potential models, methods or strategies for analyzing the CHIMGEN data}

Many models, methods and strategies can be used to analyze the CHIMGEN data. For example, GWAS can identify genetic variants associated with neuroimaging phenotypes $[4,70,71]$, multifactor dimensionality reduction and derivatives can investigate genome-wide gene-gene interactions on these phenotypes [72-74], and canonical correlation and partial least square regression analyses can uncover environmental factors associated with these phenotypes [75, 76]. Although genome-wide gene-environment interaction studies theoretically need more samples than GWAS, the CHIMGEN data can be used to investigate geneenvironment interactions on neuroimaging phenotypes with effective dimension reduction or feature selection techniques [77-79]. For example, a structured linear mixed model was recently proposed to identify candidate loci that interact with environmental variables [80]. The linkage disequilibrium score regression can estimate genetic correlations of neuroimaging phenotypes with disease-, personality- or cognition-related phenotypes [81]. Mendelian randomization and mediation analysis [82] can identify potential pathways from genes to brain to cognition. Artificial intelligence techniques, such as deep learning algorithms [83], can disclose meaningful relationships between measures from different scales.

\section{Conclusion}

As an important supplement to the research field of neuroimaging genetics, the CHIMGEN cohort can be integrated with cohorts of different ethnicities, geographic locations and socioeconomic conditions to facilitate a cross-ethnic and cross-geographic understanding of the human brain. By integrating these cohorts, we can identify the effect of ethnic factors on the brain by controlling for or stratifying by geographic and socioeconomic factors. With the same strategies, we can identify common and specific geneticneuroimaging associations in various ethnic populations. More importantly, we can identify brain-related macro- and micro-environmental factors that are common to all ethnic populations or specific to a certain ethnic population. Therefore, cross-ethnic and cross-geographic studies based on integrated cohorts would enhance our understanding of how human brains differ from each other.

Acknowledgements We thank all CHIMGEN participants for all their contributions. This work was supported in part by the National Key Research and Development Program of China (Grant No. 2018YFC1314301) and the National Natural Science Foundation of China (Grant No. 81425013) contributing to the initiation of the project.

\section{Compliance with ethical standards}

Conflict of interest The authors declare that they have no conflict of interest.

Publisher's note Springer Nature remains neutral with regard to jurisdictional claims in published maps and institutional affiliations.

Open Access This article is licensed under a Creative Commons Attribution 4.0 International License, which permits use, sharing, adaptation, distribution and reproduction in any medium or format, as long as you give appropriate credit to the original author(s) and the source, provide a link to the Creative Commons license, and indicate if changes were made. The images or other third party material in this article are included in the article's Creative Commons license, unless indicated otherwise in a credit line to the material. If material is not included in the article's Creative Commons license and your intended use is not permitted by statutory regulation or exceeds the permitted use, you will need to obtain permission directly from the copyright holder. To view a copy of this license, visit http://creativecommons. org/licenses/by/4.0/. 


\section{References}

1. Hyde LW, Bogdan R, Hariri AR. Understanding risk for psychopathology through imaging gene-environment interactions. Trends Cogn Sci. 2011;15:417-27.

2. Meyer-Lindenberg A, Weinberger DR. Intermediate phenotypes and genetic mechanisms of psychiatric disorders. Nat Rev Neurosci. 2006;7:818-27.

3. Mufford MS, Stein DJ, Dalvie S, Groenewold NA, Thompson PM, Jahanshad N. Neuroimaging genomics in psychiatry-a translational approach. Genome Med. 2017;9:102.

4. Elliott LT, Sharp K, Alfaro-Almagro F, Shi S, Miller KL, Douaud $\mathrm{G}$, et al. Genome-wide association studies of brain imaging phenotypes in UK biobank. Nature. 2018;562:210-6.

5. Bookheimer SY, Strojwas MH, Cohen MS, Saunders AM, Pericak-Vance MA, Mazziotta JC, et al. Patterns of brain activation in people at risk for alzheimer's disease. N. Engl J Med. 2000;343:450-6.

6. Reed JL, D'Ambrosio E, Marenco S, Ursini G, Zheutlin AB, Blasi $\mathrm{G}$, et al. Interaction of childhood urbanicity and variation in dopamine genes alters adult prefrontal function as measured by functional magnetic resonance imaging (fmri). PLoS ONE. 2018;13:e0195189.

7. Dadvand P, Pujol J, Macia D, Martinez-Vilavella G, BlancoHinojo L, Mortamais M, et al. The association between lifelong greenspace exposure and 3-dimensional brain magnetic resonance imaging in barcelona schoolchildren. Environ Health Perspect. 2018;126:027012.

8. Poo MM, Du JL, Ip NY, Xiong ZQ, Xu B, Tan T. China brain project: basic neuroscience, brain diseases, and brain-inspired computing. Neuron. 2016;92:591-6.

9. Hao $\mathrm{N}$, Zhang $\mathrm{HH}$. Interaction screening for ultra-high dimensional data. J Am Stat Assoc. 2014;109:1285-301.

10. Kong $\mathrm{Y}$, Li D, Fan $\mathrm{Y}, \mathrm{Lv}$ J. Interaction pursuit in highdimensional multi-response regression via distance correlation. Ann Stat. 2017;45:897-922.

11. Fan J, Lv J. Sure independence screening for ultrahigh dimensional feature space. J R Stat Soc: Ser B (Stat Methodol). 2008;70:849-911.

12. Friedman L, Glover GH. Report on a multicenter fmri quality assurance protocol. J Magn Reson Imaging. 2006;23:827-39.

13. Glover GH, Mueller BA, Turner JA, van Erp TG, Liu TT, Greve $\mathrm{DN}$, et al. Function biomedical informatics research network recommendations for prospective multicenter functional mri studies. J Magn Reson Imaging: JMRI. 2012;36:39-54.

14. Davies G, Marioni RE, Liewald DC, Hill WD, Hagenaars SP, Harris SE, et al. Genome-wide association study of cognitive functions and educational attainment in UK Biobank $(\mathrm{n}=112$ 151). Mol Psychiatry. 2016;21:758-67.

15. Trampush JW, Yang MLZ, Yu J, Knowles E, Davies G, Liewald DC, et al. GWAS meta-analysis reveals novel loci and genetic correlates for general cognitive function: a report from the cogent consortium. Mol Psychiatry. 2017;22:1651-2.

16. Howard DM, Adams MJ, Shirali M, Clarke TK, Marioni RE, Davies G, et al. Genome-wide association study of depression phenotypes in UK Biobank identifies variants in excitatory synaptic pathways. Nat Commun. 2018;9:1470.

17. Weiner MW, Veitch DP, Aisen PS, Beckett LA, Cairns NJ, Green $\mathrm{RC}$, et al. The alzheimer's disease neuroimaging initiative: a review of papers published since its inception. Alzheimer's Dement 2013;9:e111-94.

18. Weiner MW, Veitch DP, Aisen PS, Beckett LA, Cairns NJ, Cedarbaum J, et al. 2014 update of the alzheimer's disease neuroimaging initiative: a review of papers published since its inception. Alzheimer's Dement. 2015;11:e1-120.
19. Schumann G, Loth E, Banaschewski T, Barbot A, Barker G, Buchel $\mathrm{C}$, et al. The IMAGEN study: reinforcement-related behaviour in normal brain function and psychopathology. Mol Psychiatry. 2010;15:1128-39.

20. Bearden CE, Thompson PM. Emerging global initiatives in neurogenetics: the enhancing neuroimaging genetics through metaanalysis (enigma) consortium. Neuron. 2017;94:232-6.

21. Sudlow C, Gallacher J, Allen N, Beral V, Burton P, Danesh J, et al. UK Biobank: an open access resource for identifying the causes of a wide range of complex diseases of middle and old age. PLoS Med. 2015;12:e1001779.

22. Barch DM, Albaugh MD, Avenevoli S, Chang L, Clark DB, Glantz MD, et al. Demographic, physical and mental health assessments in the adolescent brain and cognitive development study: rationale and description. Developmental Cogn Neurosci. 2018;32:55-66.

23. Hughes LB, Beasley TM, Patel H, Tiwari HK, Morgan SL, Baggott JE, et al. Racial or ethnic differences in allele frequencies of single-nucleotide polymorphisms in the methylenetetrahydrofolate reductase gene and their influence on response to methotrexate in rheumatoid arthritis. Ann Rheum Dis. 2006;65:1213-8.

24. Huang T, Shu Y, Cai YD. Genetic differences among ethnic groups. BMC Genomics. 2015;16:1093.

25. Eom SY, Lim JA, Kim YD, Choi BS, Hwang MS, Park JD, et al. Allele frequencies of the single nucleotide polymorphisms related to the body burden of heavy metals in the korean population and their ethnic differences. Toxicological Res. 2016;32:195-205.

26. Martin AR, Gignoux CR, Walters RK, Wojcik GL, Neale BM, Gravel S, et al. Human demographic history impacts genetic risk prediction across diverse populations. Am J Hum Genet. 2017;100:635-49.

27. Bigdeli TB, Ripke S, Peterson RE, Trzaskowski M, Bacanu SA, Abdellaoui A, et al. Genetic effects influencing risk for major depressive disorder in china and europe. Transl Psychiatry. 2017;7:e1074.

28. Chee MW, Zheng H, Goh JO, Park D, Sutton BP. Brain structure in young and old east asians and westerners: comparisons of structural volume and cortical thickness. J Cogn Neurosci. 2011;23:1065-79.

29. Tang Y, Zhao L, Lou Y, Shi Y, Fang R, Lin X, et al. Brain structure differences between Chinese and Caucasian cohorts: a comprehensive morphometry study. Hum brain Mapp. 2018;39:2147-55.

30. Long H, Liu B, Hou B, Wang C, Li J, Qin W, et al. The long rather than the short allele of 5-httlpr predisposes han chinese to anxiety and reduced connectivity between prefrontal cortex and amygdala. Neurosci Bull. 2013;29:4-15.

31. Gu J, Kanai R. What contributes to individual differences in brain structure? Front Hum Neurosci. 2014;8:262.

32. Lenroot RK, Giedd JN. The changing impact of genes and environment on brain development during childhood and adolescence: Initial findings from a neuroimaging study of pediatric twins. Dev Psychopathol. 2008;20:1161-75.

33. den Braber A, Bohlken MM, Brouwer RM, van 't Ent D, Kanai R, Kahn RS, et al. Heritability of subcortical brain measures: a perspective for future genome-wide association studies. NeuroImage. 2013;83:98-102.

34. Heck A, Fastenrath M, Ackermann S, Auschra B, Bickel H, Coynel D, et al. Converging genetic and functional brain imaging evidence links neuronal excitability to working memory, psychiatric disease, and brain activity. Neuron. 2014;81:1203-13.

35. Walters JT, Rujescu D, Franke B, Giegling I, Vasquez AA, Hargreaves A, et al. The role of the major histocompatibility complex region in cognition and brain structure: a schizophrenia gwas follow-up. Am J Psychiatry. 2013;170:877-85. 
36. Kohli MA, Lucae S, Saemann PG, Schmidt MV, Demirkan A, Hek K, et al. The neuronal transporter gene slc6a15 confers risk to major depression. Neuron. 2011;70:252-65.

37. Rietschel M, Mattheisen M, Frank J, Treutlein J, Degenhardt F, Breuer R, et al. Genome-wide association-, replication-, and neuroimaging study implicates homer1 in the etiology of major depression. Biol Psychiatry. 2010;68:578-85.

38. Lee PH, Baker JT, Holmes AJ, Jahanshad N, Ge T, Jung JY, et al. Partitioning heritability analysis reveals a shared genetic basis of brain anatomy and schizophrenia. Mol Psychiatry. 2016;21:1680-9.

39. Jack CR Jr., Wiste HJ, Weigand SD, Knopman DS, Vemuri P, Mielke MM, et al. Age, sex, and apoe epsilon4 effects on memory, brain structure, and beta-amyloid across the adult life span. JAMA Neurol. 2015;72:511-9.

40. Gamba P, Herold M. Global mapping of human settlement: Experiences, datasets, and prospects. CRC Press, 2009.

41. Farr TG, Rosen PA, Caro E, Crippen R, Duren R, Hensley S, et al. The shuttle radar topography mission. Rev. Geophys. 2007;45: RG2004.

42. Voogt JA, Oke TR. Thermal remote sensing of urban climates. Remote Sens Environ. 2003;86:370-84.

43. Gupta P, Christopher SA, Wang J, Gehrig R, Lee Y, Kumar N. Satellite remote sensing of particulate matter and air quality assessment over global cities. Atmos Environ. 2006;40:5880-92.

44. Wilker EH, Wu C-D, McNeely E, Mostofsky E, Spengler J, Wellenius GA, et al. Green space and mortality following ischemic stroke. Environ Res. 2014;133:42-48.

45. Younan D, Tuvblad C, Li L, Wu J, Lurmann F, Franklin M, et al. Environmental determinants of aggression in adolescents: role of urban neighborhood greenspace. J Am Acad Child Adolesc Psychiatry. 2016;55:591-601.

46. Colodro-Conde L, Couvy-Duchesne B, Whitfield JB, Streit F, Gordon S, Kemper KE, et al. Association between population density and genetic risk for schizophrenia. JAMA Psychiatry. 2018;75:901-10.

47. Bloom DE, Canning D, Jamison DT. Health, wealth, and welfare. Financ Dev. 2004;41:10-5.

48. Vatovec C, Senier L, Bell M. An ecological perspective on medical care: Environmental, occupational, and public health impacts of medical supply and pharmaceutical chains. EcoHealth. 2013;10:257-67.

49. van Loo KM, Martens GJ. Genetic and environmental factors in complex neurodevelopmental disorders. Curr Genomics. 2007;8:429-44.

50. Gao W, Grewen K, Knickmeyer RC, Qiu A, Salzwedel A, Lin W, et al. A review on neuroimaging studies of genetic and environmental influences on early brain development. NeuroImage 2019;185:802-12.

51. Van Ijzendoorn MH, Bakermans-Kranenburg MJ, Belsky J, Beach S, Brody G, Dodge KA, et al. Gene-by-environment experiments: a new approach to finding the missing heritability. Nat Rev Genet. 2011;12:881.

52. Risch N, Herrell R, Lehner T, Liang KY, Eaves L, Hoh J, et al. Interaction between the serotonin transporter gene (5-httlpr), stressful life events, and risk of depression: a meta-analysis. JAMA. 2009;301:2462-71.

53. Bulik-Sullivan B, Finucane HK, Anttila V, Gusev A, Day FR, Loh PR, et al. An atlas of genetic correlations across human diseases and traits. Nat Genet. 2015;47:1236-41.

54. Maier R, Moser G, Chen GB, Ripke S, Cross-Disorder Working Group of the Psychiatric Genomics C, Coryell W, et al. Joint analysis of psychiatric disorders increases accuracy of risk prediction for schizophrenia, bipolar disorder, and major depressive disorder. Am J Hum Genet. 2015;96:283-94.

55. Mullins N, Power RA, Fisher HL, Hanscombe KB, Euesden J, Iniesta $\mathrm{R}$, et al. Polygenic interactions with environmental adversity in the aetiology of major depressive disorder. Psychological Med. 2016;46:759-70.

56. Peyrot WJ, Milaneschi Y, Abdellaoui A, Sullivan PF, Hottenga JJ, Boomsma DI, et al. Effect of polygenic risk scores on depression in childhood trauma. Br J Psychiatry. 2014;205:113-9.

57. Dunn EC, Wiste A, Radmanesh F, Almli LM, Gogarten SM, Sofer $\mathrm{T}$, et al. Genome-wide association study (GWAS) and genomewide by environment interaction study (GWEIS) of depressive symptoms in african american and hispanic/latina women. Depression Anxiety. 2016;33:265-80.

58. Green AE, Kraemer DJ, Deyoung CG, Fossella JA, Gray JR. A gene-brain-cognition pathway: prefrontal activity mediates the effect of comt on cognitive control and iq. Cereb Cortex. 2013;23:552-9.

59. Kramer AF, Bherer L, Colcombe SJ, Dong W, Greenough WT. Environmental influences on cognitive and brain plasticity during aging. J Gerontol Ser A, Biol Sci Med Sci. 2004;59:M940-57.

60. Miskowiak KW, Kjaerstad HL, Stottrup MM, Svendsen AM, Demant KM, Hoeffding LK, et al. The catechol-omethyltransferase (COMT) val158met genotype modulates working memory-related dorsolateral prefrontal response and performance in bipolar disorder. Bipolar Disord. 2017;19:214-24.

61. Wang C, Liu B, Zhang X, Cui Y, Yu C, Jiang T. Multilocus genetic profile in dopaminergic pathway modulates the striatum and working memory. Sci Rep. 2018;8:5372.

62. Xavier RM, Dungan JR, Keefe RSE, Vorderstrasse A. Polygenic signal for symptom dimensions and cognitive performance in patients with chronic schizophrenia. Schizophrenia Res Cognition. 2018;12:11-19.

63. Lederbogen F, Kirsch P, Haddad L, Streit F, Tost H, Schuch P, et al. City living and urban upbringing affect neural social stress processing in humans. Nature. 2011;474:498-501.

64. Frodl T, Janowitz D, Schmaal L, Tozzi L, Dobrowolny H, Stein DJ, et al. Childhood adversity impacts on brain subcortical structures relevant to depression. J Psychiatr Res. 2017;86: $58-65$.

65. van den Heuvel MP, Yeo BTT. A spotlight on bridging microscale and macroscale human brain architecture. Neuron. 2017;93:1248-51.

66. Liu J, Chen J, Perrone-Bizzozero N, Calhoun VD. A perspective of the cross-tissue interplay of genetics, epigenetics, and transcriptomics, and their relation to brain based phenotypes in schizophrenia. Front Genet. 2018;9:343.

67. Mariani J, Simonini MV, Palejev D, Tomasini L, Coppola G, Szekely AM, et al. Modeling human cortical development in vitro using induced pluripotent stem cells. Proc Natl Acad Sci USA. 2012;109:12770-5.

68. Ran FA, Hsu PD, Wright J, Agarwala V, Scott DA, Zhang F. Genome engineering using the crispr-cas9 system. Nat Protoc. 2013;8:2281.

69. Bogdan R, Salmeron BJ, Carey CE, Agrawal A, Calhoun VD, Garavan $\mathrm{H}$, et al. Imaging genetics and genomics in psychiatry: a critical review of progress and potential. Biol Psychiatry. 2017;82:165-75.

70. Hibar DP, Stein JL, Renteria ME, Arias-Vasquez A, Desrivieres S, Jahanshad $\mathrm{N}$, et al. Common genetic variants influence human subcortical brain structures. Nature. 2015;520:224-9.

71. Hibar DP, Adams HHH, Jahanshad N, Chauhan G, Stein JL, Hofer E, et al. Novel genetic loci associated with hippocampal volume. Nat Commun. 2017;8:13624.

72. Pan Q, Hu T, Moore JH. Epistasis, complexity, and multifactor dimensionality reduction. Methods Mol Biol. 2013;1019:465-77.

73. Hu T, Sinnott-Armstrong NA, Kiralis JW, Andrew AS, Karagas MR, Moore JH. Characterizing genetic interactions in human disease association studies using statistical epistasis networks. BMC Bioinforma. 2011;12:364. 
74. Gui J, Andrew AS, Andrews P, Nelson HM, Kelsey KT, Karagas MR, et al. A robust multifactor dimensionality reduction method for detecting gene-gene interactions with application to the genetic analysis of bladder cancer susceptibility. Ann Hum Genet. 2011;75:20-28.

75. Krishnan A, Williams LJ, McIntosh AR, Abdi H. Partial least squares (PLS) methods for neuroimaging: A tutorial and review. NeuroImage. 2011;56:455-75.

76. Smith SM, Nichols TE, Vidaurre D, Winkler AM, Behrens TE, Glasser MF, et al. A positive-negative mode of population covariation links brain connectivity, demographics and behavior. Nat Neurosci. 2015;18:1565-7.

77. Gauderman WJ, Zhang P, Morrison JL, Lewinger JP. Finding novel genes by testing $\mathrm{g} x$ e interactions in a genome-wide association study. Genet Epidemiol. 2013;37:603-13.

78. Su YR, Di CZ, Hsu L. Genetics, Epidemiology of Colorectal Cancer C. A unified powerful set-based test for sequencing data analysis of gxe interactions. Biostatistics. 2017;18:119-31.
79. Jiao S, Peters U, Berndt S, Bezieau S, Brenner H, Campbell PT, et al. Powerful set-based gene-environment interaction testing framework for complex diseases. Genet Epidemiol. 2015;39: 609-18.

80. Moore R, Casale FP, Jan Bonder M, Horta D, Consortium B, Franke L, et al. A linear mixed-model approach to study multivariate gene-environment interactions. Nat Genet. 2019;51:180-6.

81. Bulik-Sullivan BK, Loh PR, Finucane HK, Ripke S, Yang J, Schizophrenia Working Group of the Psychiatric Genomics C. et al. LD score regression distinguishes confounding from polygenicity in genome-wide association studies. Nat Genet. 2015;47:291-5.

82. Taylor DL, Jackson AU, Narisu N, Hemani G, Erdos MR, Chines $\mathrm{PS}$, et al. Integrative analysis of gene expression, DNA methylation, physiological traits, and genetic variation in human skeletal muscle. Proc Natl Acad Sci USA. 2019;116:10883-8.

83. Webb S. Deep learning for biology. Nature. 2018;554:555-7.

\section{Affiliations}

\section{Qiang $\mathrm{Xu}^{1} \cdot$ Lining Guo ${ }^{1} \cdot$ Jingliang Cheng ${ }^{2} \cdot$ Meiyun Wang ${ }^{3,4} \cdot$ Zuojun Geng $^{5} \cdot$ Wenzhen Zhu ${ }^{6} \cdot$ Bing Zhang $^{7} \cdot$} Weihua Liao ${ }^{8,9}$. Shijun Qiu ${ }^{10}$. Hui Zhang ${ }^{11} \cdot$ Xiaojun $\mathrm{Xu}^{12}$ - Yongqiang $\mathrm{Yu}^{13} \cdot \mathrm{Bo}^{13} \mathrm{Goo}^{14}$. Tong Han ${ }^{15,16}$.

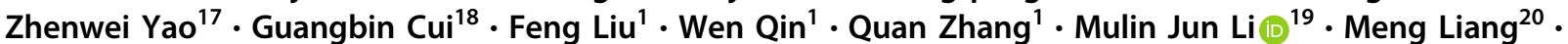

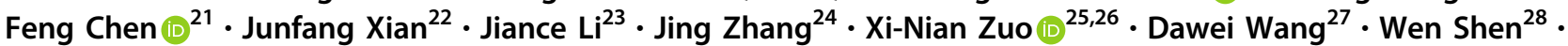
Yanwei Miao ${ }^{29}$. Fei Yuan ${ }^{30}$. Su Lui ${ }^{31,32} \cdot$ Xiaochu Zhang ${ }^{33,34} \cdot \mathrm{Kai}^{\mathrm{X}} \mathrm{Xu}^{35,36}$. Long Jiang Zhang ${ }^{37}$. Zhaoxiang Ye $\mathrm{Ye}^{38}$. Chunshui $\mathrm{Yu}^{1,39} \cdot$ for the CHIMGEN Consortium

1 Department of Radiology and Tianjin Key Laboratory of Functional Imaging, Tianjin Medical University General Hospital, 300052 Tianjin, China

2 Department of Magnetic Resonance Imaging, The First Affiliated Hospital of Zhengzhou University, 450052 Zhengzhou, China

3 Department of Radiology, Zhengzhou University People's Hospital and Henan Provincial People's Hospital, 450003 Zhengzhou, China

4 Henan Key Laboratory for Medical Imaging of Neurological Diseases, 450003 Zhengzhou, China

5 Department of Medical Imaging, The Second Hospital of Hebei Medical University, 050000 Shijiazhuang, China

6 Department of Radiology, Tongji Hospital, Tongji Medical College, Huazhong University of Science and Technology, 430030 Wuhan, China

7 Department of Radiology, Drum Tower Hospital, Medical School of Nanjing University, 210008 Nanjing, China

8 Department of Radiology, Xiangya Hospital, Central South University, 410008 Changsha, China

9 National Clinical Research Center for Geriatric Disorder, 410008 Changsha, China

10 Department of Medical Imaging, The First Affiliated Hospital of Guangzhou University of Chinese Medicine, 510405 Guangzhou, China

11 Department of Radiology, The First Hospital of Shanxi Medical University, 030001 Taiyuan, China
12 Department of Radiology, The Second Affiliated Hospital of Zhejiang University, School of Medicine, 310009 Hangzhou, China

13 Department of Radiology, The First Affiliated Hospital of Anhui Medical University, 230022 Hefei, China

14 Department of Radiology, Yantai Yuhuangding Hospital, 264000 Yantai, China

15 Department of Radiology, Tianjin Huanhu Hospital, 300350 Tianjin, China

16 Tianjin Key Laboratory of Cerebral Vascular and Neurodegenerative Diseases, 300350 Tianjin, China

17 Department of Radiology, Huashan Hosptial, Fudan University, 200040 Shanghai, China

18 Functional and Molecular Imaging Key Lab of Shaanxi Province \& Department of Radiology, Tangdu Hospital, The Military Medical University of PLA Airforce (Fourth Military Medical University), 710038 Xi'an, China

19 Collaborative Innovation Center of Tianjin for Medical Epigenetics, Tianjin Key Laboratory of Medical Epigenetics, School of Basic Medical Sciences, Tianjin Medical University, 300070 Tianjin, China

20 School of Medical Imaging, Tianjin Medical University, 300203 Tianjin, China

21 Department of Radiology, Hainan General Hospital, 570311 Haikou, China

22 Department of Radiology, Beijing Tongren Hospital, Capital Medical University, 100730 Beijing, China 
23 Department of Radiology, The First Affiliated Hospital of Wenzhou Medical University, 325000 Wenzhou, China

24 Department of Magnetic Resonance, Lanzhou University Second Hospital, 730050 Lanzhou, China

25 Department of Psychology, University of Chinese Academy of Sciences (CAS), 100049 Beijing, China

26 CAS Key Laboratory of Behavioral Science, Institute of Psychology, 100101 Beijing, China

27 Department of Radiology, Qilu Hospital of Shandong University, 250012 Jinan, China

28 Department of Radiology, Tianjin First Center Hospital, 300192 Tianjin, China

29 Department of Radiology, The First Affiliated Hospital of Dalian Medical University, 116011 Dalian, China

30 Department of Radiology, Pingjin Hospital, Logistics University of Chinese People's Armed Police Forces, 300162 Tianjin, China

31 Department of Radiology, The Center for Medical Imaging, West China Hospital of Sichuan University, 610041 Chengdu, China
32 Department of Radiology, The Second Affiliated Hospital and Yuying Children's Hospital of Wenzhou Medical University, 325000 Wenzhou, China

33 CAS Key Laboratory of Brain Function and Disease, University of Science and Technology of China, 230026 Hefei, China

34 School of Life Sciences, University of Science \& Technology of China, 230026 Hefei, China

35 Department of Radiology, The Affiliated Hospital of Xuzhou Medical University, 221006 Xuzhou, China

36 School of Medical Imaging, Xuzhou Medical University, 221004 Xuzhou, China

37 Department of Medical Imaging, Jinling Hospital, Medical School of Nanjing University, 210002 Nanjing, China

38 Department of Radiology, Tianjin Medical University Cancer Institute and Hospital, National Clinical Research Center for Cancer, Tianjin's Clinical Research Center for Cancer, Key Laboratory of Cancer Prevention and Therapy, 300060 Tianjin, China

39 CAS Center for Excellence in Brain Science and Intelligence Technology, Chinese Academy of Sciences, Shanghai 200031, China 